\title{
Analysis on Evolution Characteristics of Industry University Research Innovation Network in Guizhou Province Based on Patent
}

\author{
Yu Zhao, Nana Hu \\ Business School, Guilin University of Technology, Guilin, China
}

\begin{abstract}
Based on the industry-university-research cooperation patent data of Guizhou Province from 1986 to 2020 , this paper constructs Guizhou industry-university-research innovation network, and empirically explores the overall structural characteristics of Guizhou industry-university-research innovation network, such as network scale and network density, as well as the time evolution dynamics of nodes and cooperation intensity. It is found that the scale of industry-university-research innovation network in Guizhou Province is gradually expanding, the nodes are gradually increasing, and more cooperative groups have been formed, but the overall network is low density; Guizhou University and other universities and scientific research institutions have always occupied the central position of the network. Although enterprises are not in the core position, the intensity of cooperation with institutions is gradually increasing.
\end{abstract}

Keywords: Underdeveloped areas, Industry-university-research cooperative innovation, Innovation network evolution.

\section{Introduction}

Improving innovation competitiveness and forming an industry-university-research network of innovation cooperation among the government, enterprises, universities and research institutions will play an important role in the innovation and economic development of enterprises, regions and even countries. Guizhou province enjoys unique regional advantages and status in the construction of China-ASEAN Free Trade Area. By 2020, the GDP growth rate of Guizhou province has been among the top in China for ten consecutive years. However, it is also a less developed region in western China. In 2020, the total technological transaction volume of Guizhou province only accounts for about $0.88 \%$ of the national total. The innovation efficiency and competitiveness of the industry-university-research innovation network of Guizhou Province are lower, and the innovation development lags behind that of developed regions. Therefore, it is of great significance to study the industry-university-research innovation network in the backward regions represented by Guizhou province to improve the operating efficiency of the innovation network in the backward regions and promote regional innovation development.

At present, the existing literature mainly focuses on the formation mechanism, structural characteristics and innovation performance of industry-university-research innovation network, and there is little research on its time evolution law. The research objects also mostly focus on the research of economically developed areas, advantageous industries and so on, and pay less attention to the industry-university-research innovation network in late developing areas (Huang, 2017; Janet, 2011; Liu, 2014)[1-3]. Therefore, this paper takes this opportunity, takes Guizhou Province as the research object, constructs the industry-university-research innovation network based on the cooperative patent data of Guizhou Province, deeply explores its overall characteristics, network time evolution process and characteristics, and improves and enriches the research on the evolution law of industry-university-research cooperation network, It also provides a scientific basis for Guizhou Province and other underdeveloped areas to formulate industry-university-research innovation policies.

\section{Data Sources and Methods}

China's State Intellectual Property Office makes standardized disclosure of patent information such as joint patentees, application and publication dates, which is regarded as an important basis for the study of technological innovation achievements. By screening out institutional cooperative patents and combining with the social network analysis method, this paper can establish a patent-based industry-university-research innovation network. Based on the patent data of Guizhou Province from 1986 to 2020 released by the State Intellectual Property Office, select two or more patentees who are institutions and at least one of them applies for invention patents with an address of Guizhou, so as to reflect the cooperative innovation relationship between industry, university and research institution in Guizhou Province and establish an industry-university-research innovation network. At the same time, the social network analysis software GEPHI and UCINET are used to process, calculate and analyze the selected data to explore the characteristics of network time evolution.

\section{Results and Analysis}

\subsection{Overall Characteristics of Innovation Network in} Guizhou Province

Import all the data of cooperative patents in Guizhou Province from 1986 to 2020 into GEPHI 0.9.2, obtain the undirected network with 1427 nodes and 1361 edges, and draw the patent cooperation network diagram (omitted) by using "Fruchterman Reingild" layout. At the same time, after matrix processing, the data are imported into UCINET 6.199, and the network characteristic index values such as network diameter 12 , network average weighting 7.319 and average path length 
4.672 are calculated in the two software. It can be seen that the overall network scale of Guizhou industry-university-research innovation network is large, the participation of various subjects is high, and the network diameter reaches the stable boundary value of the network, indicating that the subjects in the network are easier to establish innovative cooperation (Bettencourt, 2009)[4]. However, it is worth noting that the overall network density is only 0.001 , that is, although the innovation network in Guizhou Province has a large scale and strong network heterogeneity, the connection strength between network subjects is low and the cooperation is loose. This paper analyzes the "Degree" (indicating the degree to which the node resides in the center of the network), "Closeness Centrality" (indicating the degree to which the node is not controlled by other actors), "Betweenness Centrality" (indicating the degree index to which the node resides between the other two actors), and "Edge Weight" (indicating the cooperation intensity of the node), which plays an important role in the analysis of the contribution of important institutions in the industry-university-research innovation network, the cooperative relationship between institutions, the control of key institutions over innovation resources and the intensity of cooperation (Liu, 2004)[5]. According to the calculation of GEPHI 0.9.2, the important nodes of Guizhou industry-university-research innovation network ranked by "Degree" (Table 1, only the top 7 are shown). It can be seen that universities and scientific research institutions play an important role in this innovation network of Guizhou Province. The most influential institution is Guizhou University. In addition, Guizhou Electric Power Test and Research Institute has the strongest control ability over resource allocation and other nodes. The proximity centrality of enterprise groups is weak, indicating that the relationship between enterprises and other nodes is tight. In addition, although Guizhou Kexin chemical and metallurgical Co., Ltd. is not in the core position of the network, the weight of the edges formed between them and Guizhou University is the largest, indicating that the number of cooperation is the largest and the strength of the cooperation relationship is larger.

Table 1: The important nodes of Guizhou industry-university-research innovation network

\begin{tabular}{|c|c|c|c|}
\hline Nodes & $\begin{array}{c}\text { Degr } \\
e e\end{array}$ & $\begin{array}{c}\text { Closenes } \\
\text { Centralit } \\
y\end{array}$ & $\begin{array}{c}\text { Betweenness } \\
\text { Centrality }\end{array}$ \\
\hline Guizhou University & 153 & 0.398638 & 224504.9 \\
\hline $\begin{array}{c}\text { Guizhou electric power test and } \\
\text { Research Institute }\end{array}$ & 41 & 0.334212 & 59324.38 \\
\hline Guizhou Normal University & 26 & 0.264512 & 32208.37 \\
\hline Guizhou Power Grid Corporation & 24 & 0.233007 & 17596.54 \\
\hline $\begin{array}{c}\text { Guizhou Academy of Tobacco } \\
\text { Science }\end{array}$ & 24 & 0.314723 & 25840.03 \\
\hline
\end{tabular}

Overall, the industry-university-research innovation network in Guizhou Province is in a stable development stage and has not formed a mature innovation network. Its network scale is large, but the cooperation density is very small, and the cooperation is relatively loose; In the network, universities and scientific research institutions occupy the core position and have strong knowledge creation ability. However, the network as a whole is still dominated by universities and scientific research institutions. Most enterprises with network power are state-owned capital, but enterprises are forming strong network cooperation and developing to the network center.

\subsection{Time Evolution of Industry-university-research Innovation Network in Guizhou Province}

Through the comparative analysis of the total number of patents and the number of industry-university-research cooperation patents in Guizhou Province, the data of cooperation patents are divided into three stages: 1986-2005, 2006-2015 and 2016-2020. In the first stage, the total number of patents is small, and the average number of cooperative patents is no more than 10; The second stage: the number of cooperative patents increased rapidly, reaching a maximum of 503 in 2016, and the innovative links between industry, university and research subjects increased rapidly; The third stage: cooperation patents decreased in 2016 compared with 2015 , and then increased year by year, but the growth rate slowed down. The data of these three stages are calculated and analyzed respectively, the undirected network diagram of the three stages is obtained, and the structural indicators such as the total number of network nodes, the number of network edges and network density of the three stages are calculated (Table 2). At the same time, combined with the central indicators of important network nodes in each stage, the evolution law of industry-university-research innovation network in Guizhou Province is revealed.

Table 2: The characteristics of innovation network in Guizhou Province from 1986 to 2020

\begin{tabular}{|c|c|c|c|}
\hline Value & $1986-2005$ & $2006-2015$ & $2016-2020$ \\
\hline Nodes & 51 & 549 & 1122 \\
\hline Connect & 29 & 539 & 982 \\
\hline Density & 0.023 & 0.004 & 0.002 \\
\hline Weighting & 2.314 & 6.707 & 5.922 \\
\hline Diameter & 2 & 11 & 13 \\
\hline Path length & 1.256 & 4.254 & 5.197 \\
\hline
\end{tabular}

The first stage is the initial formation of the industry-university-research innovation network in Guizhou Province. At this time, the innovation network generally presents the structural characteristics of "less-central nodes and more-isolated links". The number of network nodes is only 51, and the network diameter and average weighting are small, indicating that the industry-university-research innovation network is still in preliminary development at this time, but the network density is the strongest at this time, that is, the relationship between innovation subjects is close, but most of them are isolated. At this time, Guiyang Aluminum Magnesium Design and Research Institute, Guizhou Institute of chemical metallurgy and Institute of Geochemistry of Chinese Academy of sciences are the main nodes in the network. It can be seen that the preliminary industry-university-research innovation network takes scientific research institutions as the core, and universities and enterprises participate less and are in a marginal position.

In the second stage, the industry-university-research innovation network of Guizhou Province presents the characteristics of "multi-center nodes and multi-close links", which is the growth stage of the innovation network. The number of network nodes, connections and other indicators increased rapidly, and the network diameter increased more rapidly than that in the first stage, indicating that from 2006 to 2015 , the industry-university-research innovation network in Guizhou Province grew rapidly and the network has begun to 
take shape. At this time, compared with the isolated connection in the first stage, the connection between nodes has developed into dense connection, but with the addition of more new nodes, the overall network density still shows a decreasing trend. At this stage, Guizhou University has become the core node of the innovation network, and the cooperation intensity with enterprises is gradually increasing, and the participation of enterprises is also increasing. Apart from the group with Guizhou University as the cooperation center, a cooperation group with Guizhou electric power test and Research Institute and China Railway Fifth Bureau (Group) Co., Ltd. as the center has also been formed.

In the third stage, the industry-university-research innovation network of Guizhou Province has changed from rapid development to stable development, but the overall network is not mature. The innovation network still presents the characteristics of "multi center nodes and more close connections", but there are more nodes and denser connections. At this time, the decrease of network density and network average weighting indicates that there are more heterogeneous isolated connected nodes at this stage. These nodes are generally at the edge of the network and have weak connection with the core subject of the network. Although it increases the network scale, it reduces the network agglomeration and disperses the connection. Guizhou University is still at the core of the network and plays an important role in the distribution and control of network resources, but at the same time, more cooperative groups have been formed, and strong cooperative relationship groups centered on enterprises (such as China Railway Fifth Bureau Group Co., Ltd., Guizhou Kexin chemical Metallurgy Co., Ltd., Guiyang Sinochem Phosphorus Fertilizer Co. LTD).

Generally speaking, the industry-university-research innovation network in Guizhou Province is developing continuously, and the network scale, cooperation relationship and cooperation density are increasing. However, compared with the innovation networks in domestic developed regions, there are still some problems, such as low network agglomeration and weak heterogeneity of innovation subjects in the core position. The network center is still centered on universities and scientific research institutions. Enterprises do not play a leading role and do not form a mature industry-university-research innovation network with enterprises as the core, which is not conducive to the transformation of scientific and technological achievements and the improvement of innovation output efficiency of industry university research.

\section{Conclusion}

The overall scale of Guizhou industry-university-research innovation network is large, with a large number of nodes and dense connections. The network scale is at the edge of the stable range, but the overall density of the network is low and the network agglomeration is not strong. Among them, the universities represented by Guizhou University occupy the core position of the network, and the cooperative relationship is still dominated by universities and scientific research institutions. From the perspective of time evolution, the industry-university-research innovation network in Guizhou Province has developed rapidly, the number of nodes is increasing, and the scale of the network is expanding, forming a network form of "multicenter, multi node and close connection". Scientific research institutions occupy the center of the network in the early stage of the formation of the network. During the period of rapid development of the network, Guizhou University and other universities gradually become the center of the whole network, have greater power and influence, and have an impact on resource allocation and control. In the final stage, the cooperation groups formed by universities and scientific research institutions are still the center of the innovation network, but the cooperation intensity with enterprises continues to increase. From a small number of enterprises in the initial stage to a large number of state-owned enterprises in the rapid development period, and then to the formation of cooperation groups of private enterprises, the status of enterprises in the innovation network continues to increase, However, Guizhou Province has not yet formed a mature industry university research innovation system.

Recommendations: First, emphasize the role of the government. The government also plays an important role in the industry-university-research innovation network. The government can guide the cooperation between enterprises and other institutions by formulating innovation policies, certain policy tools can be used to support the establishment of collaborative innovation centers, industrial clusters and incubators, form and develop the industry-university-research innovation network, reasonably optimize the allocation of innovation resources, and improve the operation efficiency and innovation output of the network. Secondly, highlight the importance of enterprises, especially high-tech enterprises and emphasizing the role of small and medium-sized enterprises. Promote the financing innovation of large, medium and small enterprises. Finally, we should improve the openness of the network, so as to increase the heterogeneity of the network, and actively guide intermediary institutions such as financial institutions to enter the innovation network, so as to add vitality to the industry-university-research innovation network. At the same time, strengthen the relationship with enterprises or institutions in developed regions, learn from the innovative development experience of industry, university and research in developed regions, give full play to the late development advantages of underdeveloped regions in combination with their own regional characteristics, promote the healthy and stable development of innovation network, improve innovation output and improve innovation competitiveness.

\section{Acknowledgment}

We would like to thank the support by the institutional research grant \#: 41661030 of National Natural Science Foundation of China, which is called "The Dynamics of Industry-university-research Institute Innovation Networks for High-tech Enterprises in Latecomer Regions: A Comparison of Guangxi and Yunnan Province". 


\section{References}

[1] Jingjing $H$. Research on the University-industry collaborative innovation efficiency and its affecting factors [J]. Soft Science, 2017, 31(05): 38-42.

[2] Janet B, Maryann F. The mechanisms of collaboration in inventive teams: Composition, social networks, and geography [J]. Research Policy, 2011, 40(1):81-93.

[3] Guoqing L, Gang Z, Jinlong G. Innovation Network System of Industry-University-Research Institute of Equipment Manufacturing Industry in the Changjiang River Delta $[\mathrm{J}]$. Scientia Geographica Sinica, 2014, 34(09): 1051-1059.

[4] Bettencourt L, Kaiser D I, Kaur J. Scientific discovery and topological transitions in collaboration networks[J]. Journal of Informetrics, 2009, 3(3): 210-221.

[5] Jun L. An Introduction to Social Network Analysis[M]. Beijing: Social Sciences Academic Press, 2004. 